\title{
Identification of Four Chromosomal Loci Determining Obesity in a Multifactorial Mouse Model
}

\author{
Craig H. Warden, Janis S. Fisler, Susan M. Shoemaker, Ping-Zi Wen, Karen L. Svenson, \\ Mario J. Pace, and Aldons J. Lusis \\ Department of Medicine, Department of Microbiology and Molecular Genetics, and Molecular Biology Institute, University of California, \\ Los Angeles, California 90095
}

\begin{abstract}
We previously described a new mouse model for multigenic obesity, designated BSB. We now report the use of a complete linkage map approach to identify loci contributing to body fat and other traits associated with obesity in this model. Four loci exhibiting linkage with body fat, or with the weights of four different fat depots, residing on mouse chromosomes $6,7,12$, and 15, were identified and confirmed by analysis of additional BSB mice. Each of the four loci differed with respect to their effects on the percent of body fat, specific fat depots and plasma lipoproteins. The loci exhibited allele-specific, non-additive interactions. A locus for hepatic lipase activity was co-incident with the body fat and total cholesterol loci on chromosome 7 , providing a possible mechanism linking plasma lipoproteins and obesity. The chromosome 7 locus affecting body fat, total cholesterol and hepatic lipase activity was isolated in congenic strains whose donor strain regions overlap with the chromosome 7 BSB locus. These results provide candidate genes and candidate loci for the analysis of human obesity. (J. Clin. Invest. 1995. 95:1545-1552.) Key words: lipoproteins • cholesterol - obesity • genetics $\cdot$ linkage (genetics)
\end{abstract}

\section{Introduction}

Obesity is the most powerful risk factor for non-insulin-dependent diabetes mellitus, and it is also associated with hypertension, altered plasma lipoprotein profiles, coronary artery disease and increased mortality (1-3). With rare exceptions, human obesity is a multifactorial trait which exhibits a strong familial tendency (4-6). Due to its multifactorial nature, efforts to identify the genetic factors contributing to human obesity have been largely unsuccessful. However, the development of highly polymorphic markers and new statistical procedures have made it practical to analyze polygenic quantitative traits in animal models (7-10). Studies of the NOD mouse model of insulin dependent diabetes mellitus have clearly shown the power of system-

Address correspondence to Craig H. Warden, Ph.D., Department of Medicine, Division of Cardiology, UCLA, Los Angeles, CA 900951679. Phone: 310-206-0133; FAX: 310-794-7345; e-mail: warden@ biovx1.biology.ucla.edu.

Received for publication 7 September 1994 and in revised form 29 November 1994.

J. Clin. Invest.

(c) The American Society for Clinical Investigation, Inc.

0021-9738/95/04/1545/08 $\$ 2.00$

Volume 95, April 1995, 1545-1552 atic identification of the multiple genes underlying a complex disease $(11,12)$.

In the present study we have utilized a complete linkage map approach to identify the genetic loci contributing to obesity in a new multigenic mouse model for obesity, designated BSB ${ }^{1}$ $(10,13)$. In contrast to the Mendelian mouse models for obesity $\left(o b, d b, f a t, t u b, a d, A^{Y}\right)(14)$, obesity in BSB mice resembles that observed in humans with respect to its multifactorial nature. Obesity in this model is also associated with basal hyperinsulinemia, glucose intolerance, hyperlipidemia, and increased lean body mass (13), as is often seen in obese humans. Using a partial linkage map, we previously reported evidence for two chromosomal loci contributing to obesity in BSB mice (15). We have now extended the BSB cross and have constructed a complete linkage map covering all 19 autosomal chromosomes and the $\mathrm{X}$ chromosome. The results confirm the previously identified loci on mouse chromosomes 6 and 7 and reveal two additional loci on mouse chromosomes 12 and 15. Furthermore, analysis of strains congenic (16-18) for mouse chromosome 7 indicate that a gene in this region dramatically influences body fat. The interactions among the four loci are complex rather than additive, and the loci exhibit specific effects on the distribution of body fat and traits associated with obesity.

\section{Methods}

Genetic crosses. Breeding pairs of Mus musculus domesticus (C57BL/ $6 \mathrm{~J}$ ) were obtained from The Jackson Laboratory (Bar Harbor, ME) and Mus spretus were obtained from Dr. Michael Potter (SPRET/Pt, National Institutes of Health, Bethesda, MD) or from The Jackson Laboratory (SPRET/Ei, Bar Harbor, ME). A backcross was performed by crossing $\mathrm{F} 1$ females, resulting from the cross of female C57BL/6J with male $M$. spretus mice, with male $\mathrm{C} 57 \mathrm{BL} / 6 \mathrm{~J}$ mice. We have designated these backcross progeny as BSB mice. After weaning at $21 \mathrm{~d}$, mice were individually housed in plastic cages with free access to food and water throughout the experimental period. The first cross (SPRET/Pt BSB) had a mean age of $217 \pm 95 \mathrm{~d}$ and the second cross (SPRET/Ei BSB ) had a mean age of $229 \pm 72 \mathrm{~d}$ at the time of sacrifice. Mice were fed standard commercial rodent chow containing $12 \%$ of calories as fat from weaning throughout the study ( $\mathrm{N}=207$ females, 205 males $) .99$ BSB mice were maintained on a moderate fat, high cholesterol diet (Teklad 90221; Teklad Premier Laboratory Diets, Madison, WI) containing $30 \%$ of calories as fat for $5 \mathrm{wk}$ before sacrifice (19). Preliminary studies with inbred and congenic strains suggest that prolonged exposure

1. Abbreviations used in this paper: $\mathrm{BB}$, a genetic locus homozygous for C57BL/6J alleles; cM, centiMorgans; LOD, $\log _{10}$ of the likelihood of the odds ratio; Mob-1, Mob-2, Mob-3, Mob-4, genetic loci underlying multigenic obesity; $o b$, obese mouse mutation; QTL, quantitative trait locus; SB, a genetic locus heterozygous for Mus spretus and C57BL/ $6 \mathrm{~J}$ alleles; SPRET, Mus spretus; BSB, (C57BL/6J $\times$ Mus spretus) $\times$ C57BL/6J backcross mice; tub, tubby mouse mutation. 
to the high fat diets can affect body fat content. However, the mice included in this study were on the diets for no more than $5 \mathrm{wk}$, and they showed no differences in median body fat content and the range of body fat percent as compared to mice maintained on a chow diet.

Quantitative phenotype measurements. Mice were fasted for approximately $15 \mathrm{~h}$ prior to transport to the laboratory and collection of blood through the retroorbital sinus within $90 \mathrm{~s}$ of initiating ether (SPRET/ Pt BSB) or isoflurane (SPRET/Ei BSB) anesthesia at $\sim 3 \mathrm{~h}$ into the light phase of the diurnal cycle. Blood samples were collected in plasma separator tubes containing EDTA, placed on ice and centrifuged to prepare plasma. Plasma total and HDL cholesterol, triglyceride and glycerol concentrations were determined as previously described (15). Plasma hepatic lipase activity, measured only in the SPRET/Pt BSB cross, was measured using a glycerol tri $\left[{ }^{3} \mathrm{H}\right]$ oleate substrate as previously described (20). Briefly, the substrate and aliquots of preheparin plasma were incubated at $37^{\circ} \mathrm{C}$ for $60 \mathrm{~min}$, and the liberated free fatty acid radioactivity was extracted and counted. Body-mass-index was calculated as body weight $(\mathrm{g})$ divided by the square of the anal-nasal length $(\mathrm{cm})$. Three intra-abdominal fat pads, the retroperitoneal, mesenteric, and epididymal (male) or parametrial (female) fat pads were dissected, weighed and returned to the carcass. A subcutaneous fat pad laying over the outer thigh (femoral) was also dissected, weighed and returned to the carcass. Individual fat pads were not dissected in initial sacrifices of the SPRET/Pt BSB cross. Body composition, glucose levels, insulin levels, and corticosteroid levels were measured as previously described $(13,15)$.

Genotyping and data analysis. Genotyping was performed as described previously (21). Simple sequence length variants were detected by polymerase chain reaction $(8,15)$. The quantitative trait locus (QTL) mapping method was used (9). Linkage analysis of the polymorphisms found for each locus was performed with the MAPMAKER program 3.0. Levels of significance $(P)$ and age adjusted values were calculated with the StatView and SuperANOVA programs (Abacus Concepts) on the Macintosh.

Genotyping data suggest that the two substrains of Mus spretus used are genetically quite similar, since both have identical allele sizes for $>99 \%$ of simple sequence length variants. Phenotyping results also suggest that the two substrains are similar, since values for total and HDL cholesterol levels, and for other traits, were not statistically different (data not shown). Moreover, the degree of substrain similarity does not affect QTL mapping, since data can be combined from crosses involving very different strains, such as has been done for identification of a modifier of induced intestinal neoplasia in mice (22).

\section{Results}

Construction of linkage maps. The previously reported 215 BSB mice $(13,15)$ and 197 additional BSB mice were typed for a variety of traits related to obesity. To map genes contributing to obesity the mice were typed for 81 additional markers to yield a linkage map that includes a total of 148 markers covering all chromosomes except the $\mathrm{Y}$ chromosome. The map is in good agreement with previous studies (23) and is estimated to cover $>90 \%$ of the genome with an average spacing of $\sim 10$ centimorgans (cM) between markers. Thus, with the exception of a few cases, the genes underlying the obesity in BSB mice should reside within $\sim 5 \mathrm{cM}$ of a typed marker. Fig. 1 shows the linkage maps for chromosomes $6,7,12$, and 15 .

Chromosomal loci for obesity and related traits in BSB mice. We searched for genetic loci underlying quantitative traits using the MAPMAKER/QTL program (24), which calculates the strength of associations between genotypes and phenotypes as the $\log _{10}$ of the likelihood of the odds ratio (LOD) score. It has been calculated that in the mouse a LOD score of 3.3 is the threshold for statistically significant linkage (25). In the

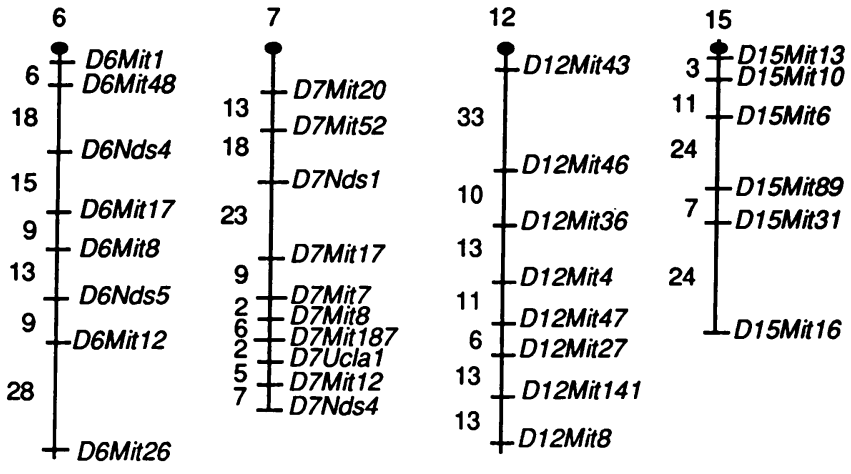

Figure 1. Linkage maps for chromosomes 6, 7, 12 and 15 for BSB mice. Linkage maps were constructed from 253 BSB mice from the cross of SPRET/Pt $\times$ C57BL/6J using the MAPMAKER 3.0 program. Distances are given as Kosambi centiMorgans. Simple sequence length polymorphism markers were taken from published maps (23). D7Uclal is a previously described restriction fragment length variant for a random mouse cDNA clone (15).

BSB cross involving SPRET/Pt, four quantitative trait loci (QTLs) exhibited significant LOD scores with respect to percent body fat or fat pad weights, with the 148 currently typed loci. We next typed an additional 197 SPRET/Ei BSB mice for genetic markers at each of the four loci. For three loci the LOD scores increased as additional animals were typed. However, for a locus on chromosome 12 the LOD score was not significantly affected by the typing of additional BSB mice. These additional mice were derived from a cross involving the inbred strain of Mus spretus derived at The Jackson Laboratory (SPRET/Ei) whereas the original cross involved a separate subline of Mus spretus (SPRET/Pt). The failure of the chromosome $12 \mathrm{QTL}$ to increase significantly may be related to different chromosome 12 alleles in the two sublines of Mus spretus used.

Comparisons of age adjusted body composition and lipoprotein levels by genotype at selected markers are presented in Table I. We previously observed D7Mit8 on distal chromosome 7 to be significantly linked to percent body fat and plasma total cholesterol in the initial cross of BSB mice (15). The locus was designated $M o b-1$, for multigenic obesity-1. The present results confirmed the existence of this locus for body fat, as the LOD score increased from 2.9 at $D 7 M i t 8$ in the previous study to 4.2 in this study. The Mob-1 locus explains $6.5 \%$ of the variance in the percent of body fat. Mice that were heterozygous for Mus spretus and C57BL/6J alleles at the D7Mit8 marker had $25 \%$ more body fat than mice that were homozygous for C57BL/6J alleles at that locus $(P<0.0003$, Table I $)$. The $M o b-1$ locus was also associated with total plasma cholesterol (LOD score 4.4) and with percent body water (LOD score 4.6). LOD scores for body mass index (LOD score 2.9 ), body weight (LOD score 2.9), and HDL cholesterol levels (LOD score 3.1) at the Mob-1 locus were just below the threshold for significance.

A second locus, on chromosome 6 near the marker D6Mitl, designated $M o b-2$, was not previously observed to be linked to percent body fat, but was linked to fat pad weight at the femoral depot (LOD score 2.7). In the expanded data set this LOD score increased to 4.8 , explaining $7.1 \%$ of the variance in femoral fat pad weight (Table I). Mice that were heterozygous at the D6Mit1 locus had about 2-fold heavier femoral fat pads than 


\begin{tabular}{|c|c|c|c|c|}
\hline & \multicolumn{2}{|c|}{ Statistics by Genotype } & \multirow[b]{2}{*}{ ANOVA } & \multirow[b]{2}{*}{ LOD } \\
\hline & BB & SB & & \\
\hline \multicolumn{5}{|l|}{ D7Mit8 } \\
\hline Body fat $(\%)$ & $\begin{array}{r}17.1 \pm 0.8 \\
n=214\end{array}$ & $\begin{array}{r}21.4 \pm 1.0 \\
n=177\end{array}$ & $P<0.0003$ & 4.2 \\
\hline Hepatic lipase activity $(\mathrm{nU} / \mathrm{ml})^{\S}$ & $\begin{array}{c}50.6 \pm 2.7 \\
n=82\end{array}$ & $\begin{array}{c}68.5 \pm 3.3 \\
n=68\end{array}$ & $P<0.0001$ & 5.1 \\
\hline Total cholesterol (mg/dl) & $\begin{array}{r}70 \pm 1.8 \\
n=187\end{array}$ & $\begin{array}{r}78 \pm 1.9 \\
n=148\end{array}$ & $P<0.0012$ & 4.4 \\
\hline HDL-cholesterol HF (mg/dl)* & $\begin{array}{c}51 \pm 3 \\
n=54\end{array}$ & $\begin{array}{l}73 \pm 5 \\
n=45\end{array}$ & $P<0.0001$ & 3.1 \\
\hline Body weight (g) & $\begin{array}{r}28 \pm 0.6 \\
n=198\end{array}$ & $\begin{array}{r}31.7 \pm 0.8 \\
n=160\end{array}$ & $P<0.0011$ & 2.9 \\
\hline Body mass index & $\begin{array}{c}0.297 \pm 0.005 \\
n=198\end{array}$ & $\begin{array}{c}0.324 \pm 0.007 \\
n=158\end{array}$ & $P<0.0015$ & 2.9 \\
\hline \multicolumn{5}{|l|}{ D6Mitl } \\
\hline Femoral fat pad (g) & $\begin{array}{c}0.47 \pm 0.04 \\
n=126\end{array}$ & $\begin{array}{c}0.95 \pm 0.09 \\
n=113\end{array}$ & $P<0.0001$ & 4.8 \\
\hline Total cholesterol (mg/dl) & $\begin{array}{r}68 \pm 1.7 \\
n=210\end{array}$ & $\begin{array}{r}77 \pm 1.7 \\
n=207\end{array}$ & $P<0.0001$ & 3.4 \\
\hline \multicolumn{5}{|l|}{ D12Mit27 } \\
\hline Body fat $(\%)^{\S}$ & $\begin{array}{r}12.1 \pm 0.8 \\
n=103\end{array}$ & $\begin{array}{c}17.4 \pm 1.2 \\
n=88\end{array}$ & $P<0.0002$ & 4.8 \\
\hline \multicolumn{5}{|l|}{ D15Mit13 } \\
\hline Mesenteric fat pad (g) & $\begin{array}{c}0.52 \pm 0.04 \\
n=141\end{array}$ & $\begin{array}{c}0.34 \pm 0.02 \\
n=151\end{array}$ & $P<0.0001$ & 3.4 \\
\hline
\end{tabular}

Data are age adjusted means \pm SE. * HF, high-fat diet (19), phenotype not affected by age and not age adjusted. ${ }^{\S}$ Based on SPRET/Pt mice only.

mice that were homozygous $\mathrm{C} 57 \mathrm{BL} / 6 \mathrm{~J}$ at that locus $(P$ $<0.0001$, Table I). The $M o b-2$ locus also contributes to total plasma cholesterol (LOD score 3.4) and exhibits suggestive linkage to percent body water (LOD score 3.2) and retroperitoneal fat, HDL-cholesterol and insulin (data not shown).

In addition to confirming the previously reported $M o b-1$ and $M o b-2$ loci, the present study has detected two novel loci, one underlying body fat percent and one modulating mesenteric fat pads. One locus resides on mouse chromosome 12 near the marker D12Mit27. It exhibits a LOD score of 4.8 for percent body fat and explains $7 \%$ of the variance (Table I and Fig. 2). Mice that were heterozygous at the D12Mit27 locus had $44 \%$ greater body fat than mice homozygous for C57BL/6J alleles at that locus $(P<0.0002$, Table $\mathrm{I})$. The chromosome 12 locus also exhibited suggestive linkage to body water and body weight (data not shown). Data on LOD scores for body fat percent of the chromosome 12 locus in the second BSB cross (SPRET/ Ei) were not included in Table I because they were neutral with respect to the final LOD score. The LOD scores for body fat percent were: both crosses combined, LOD 4.8; mice from the first cross (SPRET/Pt), LOD 4.8; mice from the second cross, LOD 0.7. The BSB linkage map for chromosome 12 is $\sim 30$ cM longer than published maps $(23,26)$. While the explanation for this is unclear, differences in genetic length should not significantly affect the peak LOD score, since LOD scores greater than 3.0 were observed at four independent loci: D12Mit4, D12Mit47, D12Mit27, and D12Mit141.

The other novel locus identified in BSB resides on mouse

chromosome 15 near D15Mit13. It exhibits a LOD score of 3.4 and explains $5.9 \%$ of the variance in mesenteric fat (Table I). Mice heterozygous at this locus had $32 \%$ smaller mesenteric fat pads than mice that were homozygous for C57BL/6J alleles $(P<0.0001$, Table $\mathrm{I})$. There was also suggestive linkage for percent body fat at the chromosome 15 locus (data not shown). We designate the chromosome 12 and 15 loci as Mob-3 and $M o b-4$, respectively.

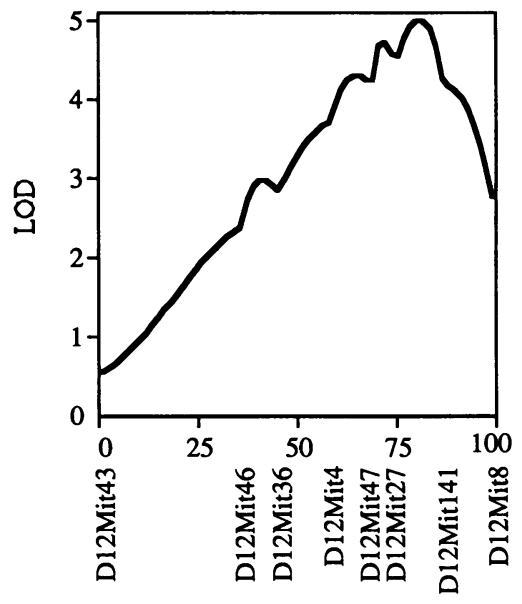

Centimorgans
Figure 2. LOD likelihood plot for the percent of body fat, adjusted for a linear effect of age, at the chromosome 12 $M o b-3$ locus. The y axis shows the LOD score calculated by the MAPMAKER/QTL program at 2-centiMorgan intervals. The $x$-axis shows the genetic distances of markers in cM. All markers are linked to each other with log likelihood scores of at least 5 . The order of these markers is supported by odds ratios of at least 100:1. 
Table II. Phenotypic Data on C57BL/10SnJ Mice and the Chromosome 7 Congenic Mice, B10.129(5M)/nSn (B10.129) and B10.C $(41 N) / S n(B 10 . C)$

\begin{tabular}{|c|c|c|c|c|c|}
\hline & \multirow[b]{2}{*}{ Background Strain C57/BL/10SnJ } & \multirow{2}{*}{$\begin{array}{c}\text { Congenic } \\
\text { B10.129 }\end{array}$} & $P$ & \multirow{2}{*}{$\begin{array}{c}\text { Congenic } \\
\text { B10.C }\end{array}$} & \multirow{2}{*}{$\frac{P}{\text { (Background vs. B10.C) }}$} \\
\hline & & & (Background vs. B10.129) & & \\
\hline Body weight (g) & $22.2 \pm 0.4$ & $19.5 \pm 0.4$ & 0.0007 & $21.3 \pm 0.8$ & NS \\
\hline AN length $(\mathrm{cm})$ & $9.2 \pm 0.1$ & $9.0 \pm 0.1$ & NS & $9.8 \pm 0.1$ & 0.005 \\
\hline Body mass index & $0.26 \pm 0.00$ & $0.24 \pm 0.01$ & 0.004 & $0.22 \pm 0.01$ & 0.001 \\
\hline Parametrial fat pad (g) & $0.53 \pm 0.04$ & $0.26 \pm 0.07$ & 0.005 & $0.38 \pm 0.07$ & NS \\
\hline Retroperitoneal fat pad (g) & $0.10 \pm 0.01$ & $0.02 \pm 0.01$ & 0.0006 & $0.03 \pm 0.01$ & 0.0003 \\
\hline Mesenteric fat pad (g) & $0.24 \pm 0.04$ & $0.25 \pm 0.02$ & NS & $0.08 \pm 0.02$ & 0.007 \\
\hline Femoral fat pad (g) & $0.27 \pm 0.02$ & $0.14 \pm 0.03$ & 0.006 & $0.04 \pm 0.02$ & $<0.0001$ \\
\hline Body fat $(\%)$ & $14.1 \pm 0.6$ & $9.2 \pm 1.4$ & 0.01 & $8.7 \pm 1.0$ & 0.003 \\
\hline Total cholesterol (mg/dl) & $60 \pm 3$ & $75 \pm 3$ & 0.01 & $67 \pm 6$ & NS \\
\hline LDL Cholesterol (mg/dl) & $4.6 \pm 1.4$ & $11.1 \pm 1.4$ & 0.01 & $7.5 \pm 3.5$ & NS \\
\hline Hepatic lipase activity (uU/ml) & $69 \pm 4$ & $47 \pm 8$ & 0.02 & $70 \pm 7$ & NS \\
\hline
\end{tabular}

Data are means \pm SE. Analyzed by Student's t-test. For C57BL/10SnJ, 10 mice were studied for body weight, AN length, and blood values; 9 mice were studied for fat pad and composition measures. Five B10.129(5M)/nSn mice were studied. For B10.C(41N)/Sn, 5 mice were studied for body composition; 4 mice were studied for blood parameters.

Linkage of hepatic lipase activity to obesity and plasma cholesterol. Human studies have revealed a relationship between hepatic lipase activity, obesity, and lipoprotein metabolism $(27,28)$. Therefore, we quantitated plasma hepatic lipase activity in the BSB mice. A QTL for hepatic lipase activity was identified on distal mouse chromosome 7 , with a peak LOD score of 5.1. This locus, which is distinct from the hepatic lipase structural gene on chromosome 9 (29), accounts for $\sim 9 \%$ of the variance in hepatic lipase activity. No other LODs $>2$ were identified for hepatic lipase activity in this cross. Mice that were heterozygous for Mus spretus and C57BL/6J alleles at the D7Mit8 marker had 35\% higher plasma hepatic lipase activity levels than mice that were homozygous for C57BL/6J alleles at that locus $(P<0.0001$, Table $\mathrm{I})$. Hepatic lipase activity in the BSB mice was strongly correlated with carcass fat (rho $=0.75)$ and with total cholesterol levels $($ rho $=0.67)$. It was also positively correlated with levels of insulin (rho $=0.56$ ) and inversely correlated with plasma corticosterone (rho = $-0.65)$. Of the four fat pads measured, hepatic lipase activity was strongly associated with the three intra-abdominal fat pads (rho $=0.68$ ), while not being significantly associated with the subcutaneous (femoral) fat pad. In contrast to $M o b-1$, none of the other obesity loci exhibited significant linkage to hepatic lipase activity (data not shown).

Isolation of obesity loci in congenic strains. We reasoned that the genetic variations influencing obesity in BSB mice may be relatively common, since many inbred strains of laboratory mice vary in body fat content and other traits related to obesity (30). Therefore, where possible, we examined previously constructed congenic strains for the chromosomal regions containing the Mob genes. Each congenic strain consists of a small chromosomal region derived from one strain that has been transferred, by selective breeding, onto the genetic background of a second strain (17). The resulting congenic strain is $98-99 \%$ identical with the background strain $(17,18)$. If the transferred chromosomal region carries one of the multigenic obesity loci, and if the donor and recipient strains carry different alleles of the obesity gene, then the congenic strain should differ from the background strain in obesity parameters.
Fortunately, several different congenic strains for loci near the Mob-1 gene on chromosome 7 were previously derived. We examined two of these, B10.129(5M)/nSn and B10.C (41N)/ $\mathrm{Sn}$. Both congenic strains are on the genetic background of strain C57BL/10SnJ. Congenic strain B10.129(5M)/nSn contains a region of chromosome 7 flanking the $H-1$ gene derived from strain 129/J (18), whereas strain B10.C(41N)/Sn contains a region of chromosome 7 flanking the $H-1$ gene derived

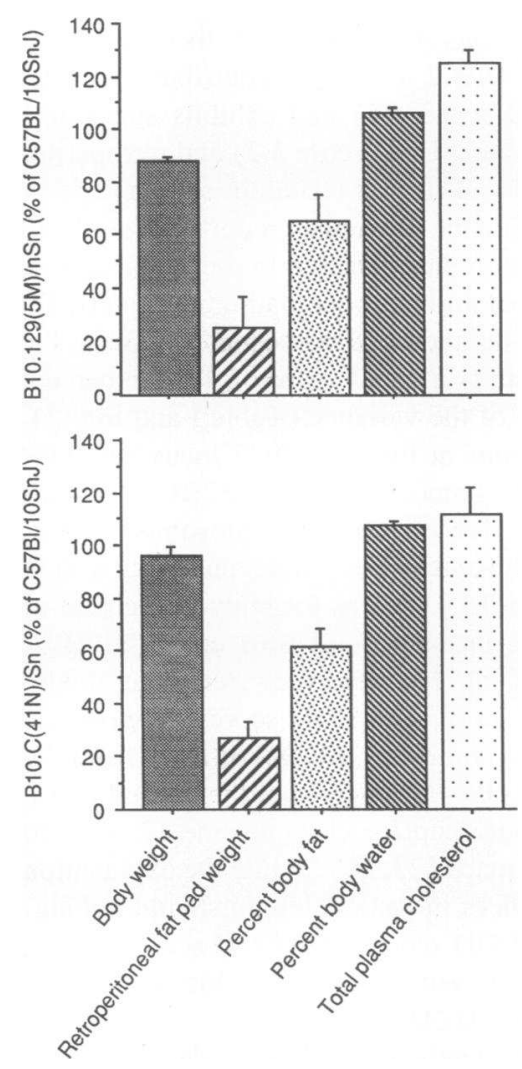

Figure 3. Effect of the $M o b-1$ gene on obesity related traits in $\mathrm{B} 10.129(5 \mathrm{M}) / \mathrm{nSn}$ and B10.C (41N)/Sn congenic strains. All animals were maintained on chow diets. Ages (in days) at sacrifice were: C57BL/10SnJ (140 n $=5,175 \mathrm{n}=5$ ), B10.C (41N)/Sn (135n $=3,149 n=2)$ and B10.129(5M)/nSn (165 $n=5$ ). Thus, all tested animals were between 135 and 175 days old at death. The C57BL/10SnJ and the B10.C (41N)/SnJ mice were derived from two litters, whereas B10.129(5M)/nSn were derived from one. Body weight, retroperitoneal fat pad, percent body fat, percent body water and total plasma cholesterol found in the congenic strains are given as percent of the C57BL/10SnJ background strain. 


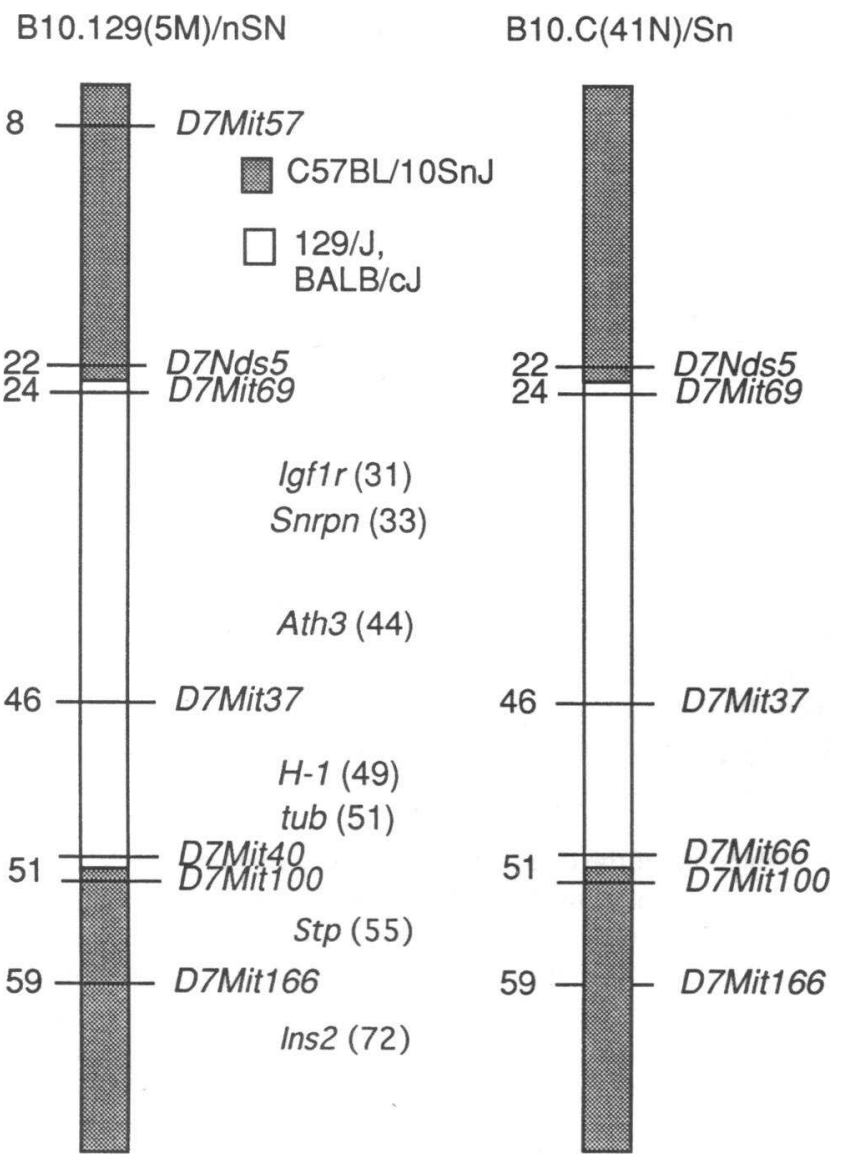

Figure 4. Molecular mapping of chromosomal breakpoints in B10.C $(41 \mathrm{~N}) / \mathrm{Sn}$ and B10.129(5M)/nSn congenic strains. Polymorphisms for D7Mit57, D7Nds5, D7Mit69, D7Mit37, D7Mit40, D7Mit100, and D7Mit166 were identified and typed in the parental and congenic strains following polymerase chain reaction (23). The distances in $\mathrm{cM}$ between markers are derived from the consensus maps of GBASE (The Jackson Laboratory). The locations of $H-l$ and various candidate genes are indicated with relative map positions indicated in parentheses.

from strain BALB/cJ (18). The two congenic strains differed from the background C57BL/10SnJ strain in several parameters related to obesity (Table II and Fig. 3). As compared to strain C57BL/10SnJ mice, the two congenic strains exhibited only $\sim 25 \%$ of the retroperitoneal fat pad weight, $\sim 60 \%$ of the body fat, and $110 \%$ of the plasma cholesterol. Total body weight and hepatic lipase activity were decreased in the B10.129(5M)/ $\mathrm{nSn}$ congenic strain. Thus, the characteristics of the locus isolated in these congenic strains is strikingly similar to the Mob1 locus identified in BSB mice.

We mapped the breakpoints between the background strainderived and donor strain-derived chromosomes. DNA was prepared from the congenic and background strains and simple sequence length polymorphisms between C57BL/10SnJ, 129/ $\mathrm{J}$ and $\mathrm{BALB} / \mathrm{cJ}$ were then identified and typed in the congenic strains. Both congenic strains contain between 27-29 cM of donor strain DNA on chromosome 7 (Fig. 4). For the B 10.129(5M)/nSn congenic, the donor $129 / \mathrm{J}$ region extends from $D 7 M i t 69$ to $D 7 M i t 40$. These results clearly suggest that D7Mit40 is proximal to D7Mit100, although recombination mapping has not separated these loci. For the B10.129(5M)/ $\mathrm{nSn}$ congenic, the congenic donor $\mathrm{BALB} / \mathrm{cJ}$ region extends

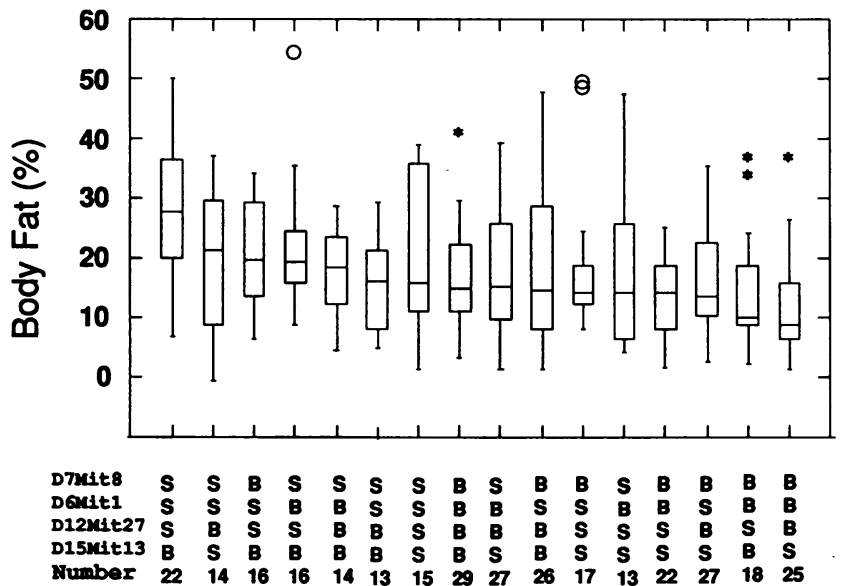

Figure 5. Genotypic interactions of $M o b$ genes in obesity. Body fat (\%) in mice with each of the 16 possible combinations of alleles at the four loci are presented as box plots. S represents mice heterozygous for Mus spretus and C57BL/6J alleles and B represents mice homozygous for C57BL/6J alleles. In this box plot, the median is marked by the center horizontal line. The upper and lower horizontal lines (the hinges) split the remaining halves in half again, covering the range from 25 to 75 percentile. The whisker lines extend from below and above the hinges to the 12.5 and 87.5 percentile. This box plot shows two different classes of outliers; outside values, plotted as asterisks, and far outside values, which are plotted as the circles (54, and SYSTSAT, Inc. Evanston, IL).

from D7Mit69 to D7Mit66, suggesting that D7Mit100 is distal to D7Mit66, although linkage maps place these markers in the reverse order (23).

\section{Discussion}

Using a complete linkage map approach, we identified four distinct loci contributing to obesity in the BSB mouse model of multigenic obesity (Mob-1, -2, -3 , and -4). The existence of three of the loci (Mob-1, -2, and -4 ) was confirmed by typing a second group of BSB mice with appropriate genetic markers, and the existence of the $M o b-1$ gene was further confirmed by analysis of congenic strains in the region of mouse chromosome 7 in which $M o b-1$ resides. This paper presents a complete and complex view of multifactorial obesity in the BSB mouse analyzed by systematic linkage mapping with a set of co-varying traits: the percent of body fat, water and protein, weight of four fat pads, body weight, body mass index, plasma insulin, glucose, corticosterone, hepatic lipase activity, and cholesterol levels. The results are consistent with the view that obesity can result from complex interactions among a variety of genes.

Fig. 5 illustrates the effects of the individual loci, as a function of genotype, on the percent body fat. We originally predicted that obesity in the BSB cross must result from the combined effects of genes from both parents, since C57BL/6J, Mus spretus, and their F1 heterozygotes are all relatively lean (15). Our current results show that Mus spretus alleles promote obesity at the chromosome 6,7 , and 12 loci, whereas C57BL/6J alleles promote obesity at the chromosome 15 locus. Furthermore, the chromosome 15 locus appears to promote obesity only if the homozygous C57BL/6J genotype occurs on a background where the chromosome 6,7 , and 12 loci are all heterozygotes. Thus, mice that are heterozygous at all four loci have median 


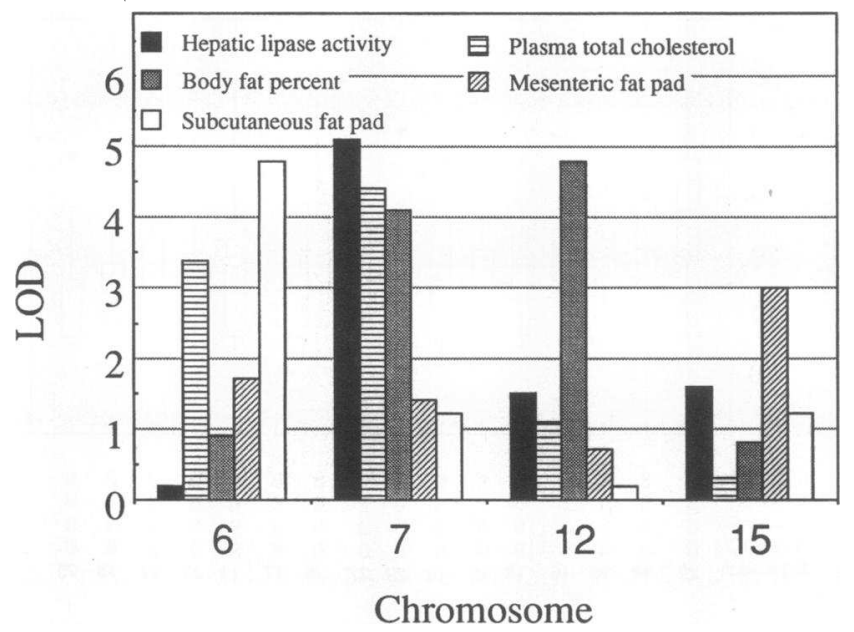

Figure 6. LOD scores for obesity and associated phenotypes at the four loci. Peak LOD scores are shown for hepatic lipase activity, plasma total cholesterol, percent body fat, mesenteric and femoral fat pads on chromosomes $6,7,12$, and 15 .

body fat of $16 \%$, whereas mice that are heterozygous on chromosomes 6, 7, and 12 but homozygous for C57BL/6J alleles on chromosome 15 have body fat of $28 \%$. Conversely, mice that are C57BL/6J homozygotes at all four loci have median body fat of $15 \%$, whereas mice with identical genotypes, except for a Mus spretus allele at the chromosome 15 locus, have median body fat of $9 \%$. In general, each of the four Mob loci does not appear to act in an additive manner with the other 3 loci.

A variety of traits, including basal hyperinsulinemia, glucose intolerance, and alterations in lipoprotein metabolism, have been associated with human obesity $(1,31,32)$. Moreover, the distribution of fat varies among individuals; thus, visceral fat in the abdomen is often associated with obesity in males, whereas subcutaneous fat is most often associated with obesity in females (33-36). It is unclear, however, whether these obesityassociated traits are the result of the effects of specific genetic factors or whether they are simply secondary to hormonal status. Nor have genetic factors contributing to the distribution of body fat been characterized. Thus, it was of interest to determine whether the Mob-1, -2, -3 , and -4 loci exert effects on any specific trait (Fig. 6). Two of the loci (chromosomes 7 [Mob1] and 12 [Mob-3]) are significant for total body fat whereas two loci (chromosomes $6[M o b-2]$ and $15[M o b-4])$ are significant for femoral and mesenteric fat, respectively. Likewise, two obesity loci are co-incident with total plasma cholesterol (chromosomes $6[M o b-2]$ and $7[M o b-1])$ and two obesity loci are not (chromosomes 12 [Mob-3] and $15[\mathrm{Mob}-4]$ ). These results suggest that obesity and cholesterol levels are correlated because two loci have pleiotropic effects on both obesity and cholesterol levels and that increased cholesterol levels are not caused by obesity per se.

Hepatic lipase activity is associated only with chromosome $7(M o b-1)$. In vivo studies have correlated an elevated hepatic lipase activity with abdominal obesity in both men and women $(27,28)$. Interestingly, this fat depot has also been correlated with an increased risk of atherosclerosis $(1,37,38)$. Our results in mice suggest a genetic link between hepatic lipase activity, intra-abdominal fat, obesity and plasma cholesterol levels.
Analyses of the chromosome 7 congenic strains are consistent with the effects of the Mob-1 gene observed in the BSB model of multigenic obesity. While the majority of the congenic donor region is proximal to the peak of the BSB QTL, the QTL and the congenic donor regions overlap for 4-5 cM. The $90 \%$ confidence interval for Mob-1 extends to the D7Mit100 locus that forms the distal border of the congenic donor regions, while the $99 \%$ confidence interval for the $M o b-1$ locus extends to the $H b b$ locus, which is $4 \mathrm{cM}$ from D7Mit100. Since $t u b$ is shown as nonrecombinant with $H b b$ and D7Mit17 in GBASE (The Jackson Laboratory, 1994), the region of overlap between the QTL and the congenics includes the tub gene. It is of interest that previous studies have shown that the tub mutation affects plasma cholesterol levels (39). Thus, although it remains possible that the Mob-1 gene and the loci identified in the congenic strains are distinct, they exhibit remarkably similar characteristics. Thus, both Mob-1 and the congenic loci have significant effects on percent body fat, plasma cholesterol levels and hepatic lipase activity. No other multigenic obesity gene in the BSB cross affects all three traits. Also, it seems unlikely that a second obesity locus should occur in a nearby region of chromosome 7.

Mapping of the donor strain chromosomal region of the $\mathrm{H}-\mathrm{I}$ congenic strains shows that it contains several candidate genes, including Snrpn, Igflr, tub, and Ath-3 (see Fig. 4). Snrpn is an imprinted gene in both humans and mice and is located in the region of mouse chromosome 7 homologous to the human Prader-Willi locus (40). Prader-Willi is a maternally imprinted disease associated with hyperphagia and obesity. The tub mutation is a single gene mutation of mice that leads to obesity and diabetes (41). The Ath-3 gene has been reported to underlie susceptibility to atherosclerosis in the AXB and BXA recombinant inbred strains (42). The insulin-like growth factor 1 receptor $(\operatorname{Ig} f \operatorname{lr})$ is also an obesity candidate gene (43). Several candidate genes are clearly outside of the donor strain region: Stp, phenol preferring sulfotransferase; Ins2, the second mouse insulin locus (44); and $\operatorname{Ig} f 2$, the locus for insulin-like growth factor 2 .

The Mob-2, -3, and -4 loci also contain candidate genes for obesity. The $M o b-2$ locus occurs near a mutation, $o b$, that causes severe obesity and diabetes in the mouse. The $o b$ gene on chromosome 6 is $\sim 4 \mathrm{cM}$ from D6Mit1. The human locus homologous to $o b$ is predicted to reside on chromosome 7q (45). Linkage analysis of 402 sib-pairs in the Québec family study of obesity has shown that the KELL blood group antigen on $7 q 33$ is linked to body-mass-index (weight $/$ height $^{2}$ ) and to the sum of six skinfold measurements $(P<0.0001)(46)$, suggesting that the human homologue of the Mob-2 locus could contribute to obesity. The $M o b-3$ locus covers a region of distal chromosome 12 that contains the gene for thyroid stimulating hormone receptor (Tshr) (Fig. 2). The peak LOD score for the $M o b-4$ locus on chromosome 15 is very near the gene for growth hormone receptor $(G h r)$. Growth hormone and IGF-1 have recently been recognized as primary agents partitioning energy between fat and lean tissue. Moreover, both basal and stimulated growth hormone concentrations are decreased in obese individuals (47-49) and are returned toward normal with weight loss $(50,51)$. On the other hand, growth hormone receptor number is elevated in obesity (43). In contrast, concentrations of insulin-like growth factor-1 (IGF-1), a primary mediator of growth hormone's effects, are elevated and IGF-1 receptors are decreased in obesity (43). 
Previously QTL mapping has been used to identify loci underlying diet-induced obesity in a cross between strains $\mathrm{SWR} / \mathrm{J}$ and $\mathrm{AKR} / \mathrm{J}(52,53)$. Whole genome mapping of this cross revealed loci on chromosomes 4,9 and 15 . It is possible that the chromosome 15 locus in the $S W R / J \times A K R / J$ cross is identical with that in BSB mice since it is located on the proximal portion of the chromosome with the peak LOD score at D15Nds2, $8.6 \mathrm{cM}$ distal to D15Mit13 (53).

The construction of congenic strains containing the various loci contributing to obesity is of primary importance to define their locations more precisely and to examine their interactions in detail. A chromosome 7 locus that may correspond to Mob$l$ was identified in two previously isolated congenic strains, but congenic strains for the chromosome 6, 12, and 15 loci are not available. Using genetic markers, it should be feasible to isolate the various BSB loci using relatively small numbers of matings (25). The availability of congenic strains will also aid in the analysis of candidate genes residing at the various loci and, if necessary, the positional cloning of novel genes.

In conclusion, the present results have revealed or confirmed the locations of four separate loci contributing to obesity in a multifactorial mouse model. Genetic analysis of the corresponding (syntenic) chromosomal regions in humans, and examination of candidate genes residing at the loci, may provide an understanding of genetic factors in human obesity.

\section{Acknowledgments}

We thank Mark Cuevas for assistance with body composition measurements and we thank John Hill and Howard Wong for measurements of HL activity.

This work was supported by National Institutes of Health grants DK-45066 and HL-42488.

\section{References}

1. Brindley, D. N., and Y. Rolland. 1989. Possible connections between stress, diabetes, obesity, hypertension and altered lipoprotein metabolism that may result in atherosclerosis. Clin. Sci. 77:453-461.

2. Brindley, D. N., B. S. McCann, R. Niaura, C. M. Stoney, and E. C. Suarez. 1993. Stress and lipoprotein metabolism: Modulators and mechanisms. Metabolism. 42:Suppl. 1:3-15.

3. Lew, E. A., and L. Garfinkel. 1979. Variations in mortality by weight among 750,000 men and women. J. Chronic Dis. 32:563-576.

4. Bouchard, C., and L. Pérusse. 1988. Heredity and body fat. Annu. Rev. Nutr. 8:259-277.

5. Bouchard, C. 1991. Current understanding of the etiology of obesity: genetic and nongenetic factors. Am. J. Clin. Nutr. 53:1561S-1565S.

6. Bouchard, C., J.-P. Després, and A. Tremblay. 1991. Genetics of obesity and human energy metabolism. Proc. Nutr. Soc. 50:139-147.

7. Love, J. M., A. M. Knight, M. A. McAleer, and J. A. Todd. 1990. Towards construction of a high resolution map of the mouse genome using PCR-analysed microsatellites. Nuc. Acids Res. 18:4123-4130.

8. Dietrich, W., H. Katz, S. E. Lincoln, H.-S. Shin, J. Friedman, N. C. Dracopoli, and E. S. Lander. 1992. A genetic map of the mouse suitable for typing intraspecific crosses. Genetics. 131:423-447.

9. Lander, E. S., and D. Botstein. 1989. Mapping Mendelian factors underlying quantitative traits using RFLP linkage maps. Genetics. 121:185-199.

10. Warden, C. H., and J. S. Fisler. 1994. Identification of genes underlying polygenic obesity in animal models. In The Genetics of Obesity. C. Bouchard, editor. CRC Press, Boca Raton, Florida. 181-197.

11. Ghosh, S., S. M. Palmer, N. R. Rodrigues, H. J. Cordell, C. M. Hearne, R. J. Cornall, J.-B. Prins, P. McShane, G. M. Lathrop, L. B. Peterson, L. S. Wicker, and J. A. Todd. 1993. Polygenic control of autoimmune diabetes in nonobese diabetic mice. Nature Genet. 4:404-409.

12. Risch, N. S. Ghosh, and J. A. Todd. 1993. Statistical evaluation of multiple-locus linkage data in experimental species and its relevance to human studies: Application to nonobese diabetic (NOD) mouse and human insulin-dependent diabetes mellitus (IDDM). Am. J. Hum. Genet. 53:702-714.
13. Fisler, J. S., C. H. Warden, M. J. Pace, and A. J. Lusis. 1993. BSB: a new mouse model of multigenic obesity. Obesity Res. 1:271-280.

14. Friedman, J. M., R. L. Leibel, and N. Bahary. 1991. Molecular mapping of obesity genes. Mamm. Genome. 1:130-144.

15. Warden, C. H., J. S. Fisler, M. J. Pace, K. L. Svenson, and A. J. Lusis. 1993. Coincidence of genetic loci for plasma cholesterol levels and obesity in a multifactorial mouse model. J. Clin. Invest. 92:773-779.

16. Mehrabian, M., J.-H. Qiao, R. Hyman, D. Ruddle, C. Laughton, and A. J. Lusis. 1993. Influence of the apoA-II gene locus on HDL levels and fatty streak development in mice. Arterioscler. Thromb. 13:1-10.

17. Bailey, D. W. 1975. Genetics of histocompatibility in mice. I. New loci and congenic lines. Immunogenet. 2:249-256.

18. Graff, R. J., and G. D. Snell. 1968. Histocompatibility genes of mice: VIII. The alleles of the $H-1$ locus. Transplantation. 6:598-617.

19. Paigen, B., D. Mitchell, K. Reue, A. Morrow, A. J. Lusis, and R. C. LeBoeuf. 1987. Ath-1, a gene determining atherosclerosis susceptibility and high density lipoprotein levels in mice. Proc. Natl. Acad. Sci. USA. 84:3763-3767.

20. Twu, J. S., A. S. Garfinkel, and M. C. Schotz. 1984. Hepatic lipase: purification and characterization. Biochim. Biophys. Acta. 792:330-337.

21. Warden, C. H., M. Mehrabian, K. He, M.-Y. Yoon, A. Diep, Y.-R. Xia, P.-Z. Wen, K. L. Svenson, R. S. Sparkes, and A. J. Lusis. 1993. Linkage mapping of 40 randomly isolated liver cDNA clones in the mouse. Genomics. 18:295-307.

22. Dietrich, W. F., E. S. Lander, J. S. Smith, A. R. Moser, K. A. Gould, C Luongo, N. Borenstein, and W. Dove. 1993. Genetic identification of Mom-1, a major modifier locus affecting Min-induced intestinal neoplasia in the mouse. Cell. 75:631-639.

23. Dietrich, W. F., J. C. Miller, R. G. Steen, M. Merchant, D. Damron, R. Nahf, A. Gross, D. C. Joyce, M. Wessel, R. D. Dredge, A. Marquis, L. D. Stein, N. Goodman, D. C. Page, and E. S. Lander. 1994. A genetic map of the mouse with 4,006 simple sequence length polymorphisms. Nature Genet. 7:220-245.

24. Lander, E. S., P. Green, J. Abrahamson, A. Barlow, M. J. Daly, S. E. Lincoln, and L. Newburg. 1987. MAPMAKER: an interactive computer package for constructing primary genetic linkage maps of experimental and natural populations. Genomics. 1:174-181

25. Lander, E. S. and N. J. Schork. 1994. Genetic dissection of complex traits. Science (Wash. DC). 265:2037-2048.

26. Copeland, N. G., N. A. Jenkins, D. J. Gilbert, J. T. Eppig, L. J. Maltais, J. C. Miller, W. F. Dietrich, A. Weaver, S. E. Lincoln, R. G. Steen, L. D. Stein, J. H. Nadeau, and E. S. Lander. 1993. A genetic linkage map of the mouse: Current applications and future prospects. Science (Wash. DC). 262:57-66.

27. Després, J.-P., S. Ferland, S. Moorjani, A. Nadeau, A. Tremblay, P. J. Lupien, G. Thériault, and C. Bouchard. 1989. Role of hepatic-triglyceride lipase activity in the association between intra-abdominal fat and plasma HDL cholesterol in obese women. Arteriosclerosis. 9:485-492.

28. Katzel, L. I., P. J. Coon, M. J. Busby, S. O. Gottlieb, R. M. Krauss, and A. P. Goldberg. 1992. Reduced HDL2 cholesterol subspecies and elevated postheparin hepatic lipase activity in older men with abdominal obesity and asymptomatic myocardial infarction. Arterioscler. Thromb. 12:814-823.

29. Warden, C. H., R. C. Davis, M.-Y. Yoon, D. Y. Hui, K. L. Svenson, Y.-R. Xia, K.-Y. He, and A. J. Lusis. 1993. Chromosomal assignment of lipolytic enzymes in the mouse: pancreatic lipase, colipase, hormone sensitive lipase, hepatic lipase, and carboxyl ester lipase. J. Lipid Res. 34:1451-1455.

30. West, D. B., C. N. Boozer, D. L. Moody, and R. L. Atkinson. 1992. Dietary obesity in nine inbred mouse strains. Am. J. Physiol. Regul. Integr. Comp. Physiol. 262:R1025-R1032.

31. Larsson, B., P. Björntorp, and G. Tibblin. 1981. The health consequences of moderate obesity. Int. J. Obes. 5:97.

32. Kissebah, A. H., D. S. Freedman, and A. N. Peiris. 1989. Health risks of obesity. Med. Clin. North Am. 73:111-138.

33. Seidell, J. C., M. Cigolini, J. Charzewska, B.-M. Ellsinger, P. Björntorp, J. G. A. J. Hautvast, and W. Szostak. 1991. Fat distribution and gender differences in serum lipids in men and women from four European communities. Atherosclerosis. $87: 203-210$.

34. Larsson, B., C. Bengtsson, P. Björntorp, L. Lapidus, L. Sjöström, K. Svärdsudd, G. Tibblin, H. Wedel, L. Welin, and L. Wilhelmsen. 1992. Is abdominal body fat distribution a major explanation for the sex difference in the incidence of myocardial infarction? Am. J. Epidemiol. 135:266-273.

35. Bouchard, C., L. Pérusse, C. Leblanc, A. Tremblay, and G. Thériault 1988. Inheritance of the amount and distribution of human body fat. Int. J. Obes. 12:205-215.

36. Després, J.-P., S. Moorjani, P. J. Lupien, A. Tremblay, A. Nadeau, and C. Bouchard. 1990. Regional distribution of body fat, plasma lipoproteins, and cardiovascular disease. Arteriosclerosis. 10:497-511.

37. Björntorp, P. 1990. Portal adipose tissue as a generator of risk factors for cardiovascular disease and diabetes. Arteriosclerosis. 10:493-496.

38. Björntorp, P. 1991. Visceral fat accumulation: the missing link between psychosocial factors and cardiovascular disease? J. Intern. Med. 230:195-201.

39. Nishina, P. M., S. Lowe, J. Wang, and B. Paigen. 1994. Characterization of plasma lipids in genetically obese mice: the mutants Obese, Diabetes, Fat, Tubby and Lethal Yellow. Metabolism. 43:549-553. 
40. Leff, S. E., C. I. Brannan, M. L. Reed, T. Ozcelik, U. Francke, N. G. Copeland, and N. A. Jenkins. 1992. Maternal imprinting of the mouse Snrpn gene and conserved linkage homology with the human Prader-Willi syndrome region. Nature Genet. 2:259-264.

41. Coleman, D. L., and E. M. Eicher. 1990. Fat (fat) and tubby (tub): Two autosomal recessive mutations causing obesity syndromes in the mouse. J. Hered. 81:424-427.

42. Stewart-Phillips, J. L., J. Lough, and E. Skamene. 1989. Ath-3, a new gene for atherosclerosis in the mouse. Clin. Invest. Med. 12:121-126.

43. Hochberg, Z., P. Hertz, C. Varda, S. Ish-Shalom, D. Yeshuran, M. B. H. Youdim, and T. Amit. 1992. The distal axis of growth hormone (GH) in nutritional disorders: GH binding protein, insulin-like growth factor-1 (IGF-1) and IGH-1 receptors in obesity and anorexia nervosa. Metabolism. 41:106-112.

44. Jones, J. M., M. H. Meisler, M. F. Seldin, B. K. Lee, and E. M. Eicher. 1992. Localization of insulin-2 (Ins-2) and the obesity mutant tubby (tub) to distinct regions of mouse chromosome 7. Genomics. 14:197-199.

45. O'Brien, S. J., J. E. Womack, L. A. Lyons, K. J. Moore, N. A. Jenkins, and N. G. Copeland. 1993. Anchored reference loci for comparative genome mapping in mammals. Nature Genet. 3:103-112.

46. Borecki, I. B., T. Rice, L. Pérusse, C. Bouchard, and D. C. Rao. 1994. An exploratory investigation of genetic linkage with body composition and fatness phenotypes: The Québec family study. Obesity Res. 2:213-219.
47. Glass, A. R., K. D. Burman, K. T. Dahms, and T. M. Boehm. 1981. Endocrine function in human obesity. Metabolism. 30:89-104.

48. Kopelman, P. G., K. Noonan, R. Goulton, and A. J. Forest. 1985. Impaired growth hormone response to growth hormone releasing factor and insulin-hypoglycemia in obesity. Clin. Endocrinol. (Oxf). 23:87-94.

49. Mårin, P., R. Rosmond, B.-A. Bengtsson, C. Gustafsson, G. Holm, and P. Björntorp. 1994. Growth hormone secretion after testosterone administration to men with visceral obesity. Obesity Res. 2:263-270.

50. Ball, M. F., A. Z. El-Khodary, and J. I. Canary. 1972. Growth hormone response in the thinned obese. J. Clin. Endocrinol. Metab. 34:498-511.

51. Crockford, P. M., and P. A. Salmon. 1970. Hormones and obesity: changes in insulin and growth hormone secretion following surgically induced weight loss. Can. Med. Assoc. J. 103:147-150.

52. West, D. B. J. Waguespack, B. York, J. Goudey-Lefevre, and R. A. Price. 1994. Genetics of dietary obesity in AKR/J $\times$ SWR/J mice: segregation of the trait and identification of a linked locus on Chromosome 4. Mamm. Genome. 5:546-552.

53. West, D. B., J. Goudey-Lefevre, B. York, and G. E. Truett. 1994. Dietary obesity linked to genetic loci on chromosomes 9 and 15 in a polygenic mouse model. J. Clin. Invest. 94:1410-1416.

54. Tukey, J. W. 1977. Exploratory data analysis. Addison-Wesley, Reading, Mass. 